Mots. Les langages du politique

\title{
Le gaélique comme marqueur symbolique du territoire en ville de Belfast
}

Gaelic as a symbolic territory marker in Belfast

El galeico como marcador simbólico del territorio en la ciudad de Belfast

\section{Viviane Müller}

\section{(2) OpenEdition}

Journals

\section{Édition électronique}

URL : https://journals.openedition.org/mots/3973

DOI : $10.4000 /$ mots.3973

ISSN : 1960-6001

Éditeur

ENS Éditions

Édition imprimée

Date de publication : 1 mars 2004

Pagination : 13-26

ISBN : 2-84788-056-9

ISSN : 0243-6450

\section{Référence électronique}

Viviane Müller, «Le gaélique comme marqueur symbolique du territoire en ville de Belfast », Mots. Les langages du politique [En ligne], 74 | 2004, mis en ligne le 24 avril 2008, consulté le 22 avril 2022. URL: http://journals.openedition.org/mots/3973 ; DOl : https://doi.org/10.4000/mots.3973

\section{(c) ENS Éditions}




\title{
Le gaélique comme marqueur symbolique du territoire en ville de Belfast
}

\begin{abstract}
À la fin du $20^{\mathrm{e}}$ siècle, environ $10 \%$ de la population ${ }^{2}$ de la province d'Irlande du Nord possède quelque connaissance du gaélique - parlé, écrit et/ou lu. Cette communauté langagière est composée de ceux qui ont appris le gaélique à l'école secondaire, puis éventuellement à l'université, et d'enfants qui ont été élevés dans des écoles d'immersion en gaélique.

Cette compétence linguistique est le produit d'une résurrection récente. Aucune des communautés résiduelles de locuteurs natifs (gaeltacht), présentes dans la province constituée lors de la partition de l'ile ${ }^{3}$ en 1922, n'a survécu. C'est au cours des années 1970, alors que les nationalistes se soulèvent en faveur de la réforme des droits civiques de la population nord-irlandaise, que se développe l'activisme linguistique pour la restauration du gaélique. La capitale de la province, Belfast, accueille le premier groupuscule de militants qui instaure un néo-gaeltacht dans une rue du district ouest en 1971. Ces quelques familles ouvrent deux ans plus tard dans la même rue une première école gaélique sauvage (bunscoil) pour éduquer leurs sept enfants. En 1997 cette école compte près de 400 élèves et reçoit un financement public de $500000 £$. Il existe alors par ailleurs 29 crèches, 12 écoles primaires et 2 écoles secondaires gaéliques en Irlande du Nord. Elles comptent plus de 2500 élèves dont la langue première est l'anglais et qui suivent un programme d'immersion en gaélique ayant pour objectif de les rendre bilingues ${ }^{4}$.

Cette croissance reflète celle du mouvement militant qui l'a instiguée, c'est-à-dire du groupe de locuteurs celtophones qui sont engagés activement
\end{abstract}

1. Université de Neuchâtel, Institut d'ethnologie.

2. Soit approximativement 142000 personnes, selon le recensement de 1991.

3. Créant au sud la République d'Irlande, ou Éire, et au nord la province britannique d'Irlande du Nord, ou Ulster.

4. Les données présentées ici ont été recueillies en 1997, dans le cadre d'une recherche effectuée pour les instituts d'ethnologie et de linguistique appliquée de l'Université de Neuchâtel. 
dans la promotion de la langue gaélique, d'une part, et dans la définition de la signification idéologique que représente son évolution, d'autre part. Les militants considèrent les écoles gaéliques comme le mode de transmission idéal de l'identité irlandaise et leur développement comme l'objectif principal de leur mouvement.

L'évolution de ces écoles gaéliques est en outre représentative de la dynamique de l'interaction entre l'instance dirigeante et la communauté dont est issu le mouvement linguistique, ainsi que de l'attitude du gouvernement et de ses institutions à l'égard de la langue minoritaire celtique de la province. La langue gaélique est officiellement reconnue comme faisant partie de l'héritage culturel de la province depuis 1985, à savoir depuis l'accord anglo-irlandais au cours duquel le gouvernement britannique s'est engagé à traiter équitablement les droits, ce qui comprend les droits culturels, ressortissant aux deux communautés catholique et protestante de la province divisée. À la suite de cette déclaration politique, diverses institutions ont été fondées dans le but de promouvoir toute initiative favorisant les relations intercommunautaires et la connaissance du patrimoine culturel de la province, dont fait partie le gaélique.

Cet article traite particulièrement de l'utilisation du gaélique en tant que marqueur symbolique du territoire dans ce contexte. Il est question de la visibilité et de l'utilisation symbolique de cette langue en ville de Belfast, ce qui est illustré par une controverse survenue lors du retrait d'enseignes bilingues (gaélique-anglais) dans le quartier dit neutre de la capitale.

\section{Configuration territoriale}

La division politique et culturelle de la ville de Belfast, à l'image de l'ensemble de la province, est inscrite dans son territoire. À cette territorialisation concrète, qui résulte essentiellement de valeurs et pratiques développées dans l'histoire récente et violente de l'Irlande du Nord, est conjugué un système de représentations relatif à l'espace. La présentation de cette configuration territoriale est un préalable nécessaire à la compréhension de la violence symbolique que peut véhiculer toute présence du gaélique et, par conséquent, à l'interprétation des discours qui accompagnent sa mobilisation comme ressource symbolique . $^{5}$.

5. Selon l'approche situationaliste, décrire les champs de saillance permet de dégager la façon dont une langue, interprétée comme un attribut culturel, devient une ressource symbolique. Voir P. Poutignat, J. Streiff-Fenart (1995, p. 182). 


\section{Les origines}

Dès la constitution de la province, les communautés s'organisent en quartiers de résidence autour des institutions représentant leurs confessions. La population catholique manifeste le besoin de maintenir une identité corporative face à l'establishment protestant en créant des quartiers résidentiels centrés autour des églises et des écoles religieuses, instaurant par là de véritables paroisses administratives. Dès l'âge de cinq ans, chaque citoyen reçoit alors une éducation différenciée selon sa confession ${ }^{6}$, acquérant notamment une interprétation variable de l'histoire. C'est en 1978 que la House of Lords accepte le principe d'intégration de l'instruction, soit le mélange des confessions dans les écoles. L'opposition farouche à cette réforme en Ulster a comme conséquence de renforcer l'homogénéité des groupements résidentiels autour des écoles de telle ou telle confession. Toute tentative du gouvernement de Stormont de pallier ce phénomène rencontre les protestations du clergé catholique, pour qui répartir la population est une tentative de diluer son influence, de l'absorber notamment par le biais scandaleux de l'exogamie.

Par ailleurs, le regroupement de la population catholique permet un système simplifié de contrôle politique. Le gouvernement adopte un découpage préférentiel des districts politiques: la ville de Londonderry compte, par exemple, une population à $33 \%$ protestante et $60 \%$ catholique, représentée dans les années 1970 par 12 conseillers protestants et 8 catholiques. Les partis au pouvoir, s'accommodant d'abord de la situation en vertu des avantages indéniables qu'elle leur apporte, en viennent à la renforcer par le biais d'impressionnants déplacements de population. Les ghettos se cristallisent progressivement.

Lors du déclenchement des émeutes dans les années 1970, les foyers isolés au sein d'un quartier de confession opposée font l'objet des actes de vandalisme les plus violents et de menaces continuelles. C'est seulement parmi les siens qu'une famille trouve un semblant de sécurité et de protection collective. Les regroupements de population s'intensifient considérablement pendant les émeutes. Les enclaves catholiques des deux villes principales s'associent sous le contrôle de commandos de vigilance armés, assurant la défense de la population et consolidant les frontières résidentielles, au point d'en interdire l'accès aux forces de l'ordre: la police ne pénètre plus les quartiers enflammés du

6. R. B. Lepage et A. Tabouret-Keller mentionnent d'ailleurs ce système éducatif pour illustrer ce qui véhicule le maintien des frontières culturelles et ethniques (1985, p. 234). 
Bogside à Londonderry ou de Fall's Road à Belfast, alors sous l'égide de l'IRA (Irish Republican Army). Lors du débarquement des troupes militaires britanniques dans la province, les émeutes redoublent. L'UDR (Ulster Defence Regiment) répond par la violence et renforce la défense de ses territoires, tandis que les paramilitaires protestants, en réaction à l'efficacité relative des interventions militaires du gouvernement, instaurent à leur tour des No-Go areas, contrôlées par les soldats de l'UDR. Face à la répression militaire d'une part et d'autre part sous l'effet du sentiment grandissant d'absence de protection efficace par les forces de l'ordre officielles, chaque communauté organise son propre réseau sur un territoire de mieux en mieux défini.

Le développement infernal d'un tel contexte d'instabilité, de violence, de méfiance endémique, fait resurgir la valeur fondamentalement sécurisante du territoire. La propriété devient alors sacrée: elle ne saurait être profanée en aucune façon, comme l'illustrent les émeutes que provoquent les parades orangistes $^{7}$ qui s'aventurent dans les quartiers catholiques depuis quelques années. Ces défilés sont vécus comme une ingérence intolérable dans la vie de la communauté, une invasion dangereuse de son territoire, une mise en péril de la sécurité que celui-ci représente. Ce sentiment est naturellement renforcé du fait que ces défilés à caractère rituel célèbrent la victoire d'un roi et de son armée venus d'outre-mer envahir la terre des catholiques. Ainsi, le sentiment d'appartenance à la communauté, strictement délimitée, est devenu intense. Le lieu de résidence est devenu l'un des éléments qui permet cette identification. La dichotomisation ethnique, qui a conduit à l'attribution des labels religieux aux deux groupes en présence, est littéralement inscrite dans l'espace.

\section{La capitale}

À Belfast, les quartiers (areas, districts, sectors, neighbourhoods) catholiques et protestants sont strictement délimités. Cette configuration correspond à deux réseaux sociaux distincts ${ }^{8}$, mutuellement exclusifs. Très concrètement, cela se traduit par une restriction de la mobilité des individus. Il existe un réseau de bus et taxis publics, distincts par leur couleur, pour chaque sec-

7. L'Ordre d'Orange est une franc-maçonnerie protestante créée en 1785 et qui compte aujourd'hui quelque cinquante-mille partisans, qu'il convient de considérer comme des loyalistes extrémistes. Cet ordre tient son nom du roi Guillaume d'Orange, vainqueur sur Jacques II le Catholique en 1690.

8. «Un réseau social se définit par des contacts, des interactions et des transactions entre les personnes. Il implique des relations relativement stables entre les individus, relations comportant des rituels, des échanges d'informations, de biens ou de services» G. Lüdi, B. Py (1995, p. 21. Voir également synthèse de la théorie des réseaux, p. 104). 
teur. Les chauffeurs de taxis individualisés, quant à eux, refusent simplement d'effectuer des courses dans ce qu'ils considèrent être les wrong areas. De même, on ne trouve le journal An Phoblacht, d'orientation républicaine, que dans le quartier ouest, où le Newsletter unioniste n'est par contre jamais disponible. Il existe bien un entre-deux : le centre-ville. Constitué de la zone commerciale d'une part et du quartier universitaire d'autre part, il s'agit d'un lieu où résident essentiellement des étrangers, étudiants ou immigrés, tels que Chinois, Italiens et Pakistanais, une zone largement considérée comme indéfinissable.

À cela s'ajoute une topographie sociale: le sud de la ville est le territoire des nantis, où fleurissent jardins soignés et propriétés luxueuses. Il est situé à la sortie de la ville, loin des remparts que forment les collines et la mer. Cette détermination géodésique parait symboliser une capacité objective de mobilité physique, le luxe de disposer d'une ouverture sur le monde. Le nord-ouest de la ville, par contre, avec sa configuration morcelée et acculé contre la falaise bordant l'estuaire, est le domaine de la working class. Ces quartiers ont été la scène des émeutes les plus sanglantes au cours des trente dernières années et sont toujours le terrain privilégié d'actes de vandalisme de toutes sortes, notamment dans les rues-frontières, points d'interface ethnique extrêmement sensibles. C'est entre les rues adjacentes de Fall's Road et de Shankill Road qu'ont éclaté les premières émeutes au lendemain du soulèvement de Londonderry en aout $1969^{9}$. Une centaine de maisons ont alors été incendiées dans Fall's Road en l'espace d'un mois: les Shankill kids chassaient les habitants de leurs foyers, qu'ils brulaient de façon systématique. Les barricades ont rapidement été dressées. Aujourd'hui, ces rues sont divisées par un mur de cinq mètres, muni de barbelés, qui porte le triste nom de Peace Line. Une trentaine de checkpoints, construits pendant les années les plus agitées, remplissent une fonction similaire dans toute la ville: disposés à l'entrée des divers quartiers, ces postes de police militarisés, bâtiments en béton munis de caméras de surveillance et de barbelés, bien que vides pour la plupart aujourd'hui, contribuent néanmoins à marquer le territoire. À ces postes de contrôle correspond un système de surveillance aérien: l'omniprésence d'hélicoptères au-dessus de certains quartiers forme également un balisage sonore de l'espace.

9. Le soulèvement catholique réprimé violemment en ce fameux «Bloody Sunday» de 1969 est communément regardé comme le coup d'envoi des troubles qui déchirent la province depuis lors. Les affrontements entre partisans et opposants de l'union au RoyaumeUni ont causé la mort de près de 4000 personnes et d'innombrables blessés en trente ans de guerre civile. 
C'est dans ces circonstances que le lieu de résidence, le nom de famille, ainsi que l'accent, réputé légèrement différent selon les communautés, sont devenus semblablement attributifs, catégorisants.

\section{Appropriation symbolique du territoire et gaélique}

Dans un tel contexte, tout élément approprié devient hypersignifiant et est exposé pour contribuer à la délimitation symbolique du territoire. Belfast est ainsi constellé d'emblèmes ${ }^{10}$ représentant chaque communauté. Les murs peints qui ornent la majorité des quartiers résidentiels de la ville constituent notamment un véritable réseau de sites emblématiques ${ }^{11}$. Ces gigantesques fresques font l'objet de concours internes aux quartiers et sont remplacées périodiquement, formant ainsi un reflet pictural de la dynamique sociale et politique.

La langue gaélique joue un rôle déterminant sur cette scène symbolique: dans cette logique exclusive rapportée au territoire, elle contribue à la mise en relief de la communauté catholique. La combinaison identifiant politique, culture et langue se traduit par une floraison du gaélique dans les quartiers catholiques et nationalistes à partir des années 1980. C'est alors qu'apparaissent également les panneaux de rue bilingues désignant clairement le territoire des catholiques les plus dévoués à la cause culturelle ainsi que le gaélique dans les peintures murales républicaines, érigées en représentation sans équivoque d'une frontière idéologique.

Le quartier ouest de Belfast, qui contient l'une des plus larges communautés catholiques de la province, est le siège de la concentration la plus dense des écoles gaéliques et de certains lieux d'exposition privilégiés de la langue. Le long de Fall's Road se succèdent divers magasins et pubs aux enseignes gaéliques ou en caractères singeant l'ancien alphabet celtique. Le plus frappant de par sa proximité du centre-ville est indubitablement Siopa na hEalainne, le magasin à façade multicolore du parti républicain Sinn Féin (littéralement «nous-mêmes»). Un peu plus loin, le gigantesque cimetière de Milltown, où se trouvent enterrés les martyrs ${ }^{12}$ de la communauté catholique, parmi des cen-

10. «Signes sensibles qui fonctionnent comme expression concrète et affectivement valorisée de l'identité» P. Centlivres (1986, p. 98). Rappelons que tout symbole est une image fondamentale qui se base sur des conventions interprétatives et que le lien qui l'unit à son signifié repose sur une relation d'analogie. Voir P. Moliner (1996, p. 122-128).

11. A.-M. Losonczy (1997, p. 1). Description détaillée de ce réseau dans Müller (1999).

12. Dont notamment le fameux Bobby Sands, première victime de la grève de la faim du pénitencier de Long Kesh en 1981 : le gouvernement de la Dame de Fer, Mme Thatcher, n'a 
taines de tombes dont certaines datent du $18^{\mathrm{e}}$ siècle, est un des lieux privilégiés de visibilité du gaélique. De même, la Gaelic Athletic Association, située aux confins du quartier, présente un bâtiment dont les enseignes sont exclusivement en gaélique, ce qui reflète sa fonction identitaire intrinsèque due au type de sports qu'on y pratique et à son association passée avec l'Église catholique pour promouvoir la langue.

Avant tout, le quartier ouest loge les deux hauts lieux de rencontre celtophone que sont Cumann Chluainn Ard et le Culturlann ${ }^{13}$. Pour reprendre la terminologie de R. B. Lepage et A. Tabouret-Keller, il peut être considéré que la langue inscrite dans un tel contexte - un groupe de résidence localisé, relativement restreint et à mobilité déterminée - a acquis un statut iconique et que son emploi est totémique ${ }^{14}$. Il est indéniable que la cristallisation territoriale des communautés concernées est déterminante face aux significations multiples que peut incarner la langue, et tout particulièrement sa fonction emblématique. La visibilité du gaélique, variable selon les univers de la ville, correspond en outre à un comportement langagier. Les lieux privilégiés, mentionnés plus haut, sont les uniques espaces publics celtophones de Belfast. De façon décroissante, l'on trouvera ensuite certains pubs, lieux de socialisation par excellence, à nouveau principalement situés dans le quartier ouest, où les formules de salutations standard en gaélique (Dia duit! Cad é mar ata tu?/Bonjour! ça va?) sont les conditions d'entrée; puis d'autres cafés et magasins, où elles sont d'usage mais non prescriptives; et ceci jusqu'aux confins du centre-ville où trois bars, connus pour leurs concerts de musique traditionnelle irlandaise, offrent aux locuteurs celtophones les derniers lieux de pratique langagière détendue.

Ces circonstances permettent de considérer le gaélique comme une ressource symbolique importante qui contribue au balisage du territoire. Comme le remarque J.-W. Lapierre, toute langue peut être envisagée comme une «res-

pas accepté d'accorder le statut de prisonnier politique aux mutins républicains, dont dix périrent.

13. Le premier est le club de la Gaelic League de Belfast depuis 1936: son enseigne impressionnante jouxte un drapeau irlandais et orne un mur peint en vert éclatant. C'est, comme on dit, an béar is Gaeli in Uladh (le bar le plus gaélique d'Ulster). Le second est le centre culturel et linguistique irlandais, inauguré en 1991 dans une vieille église presbytérienne, qui centralise toutes les activités en faveur du gaélique: l'hebdomadaire Lá, seul journal celtophone de la province, des ateliers de théâtre, une radio demeurée longtemps clandestine, une librairie, une dizaine de salles aménagées en classes pour l'unique école secondaire de la ville (Meanscoil Feirste) et les bureaux de Gaeloiliuint, association volontariste vouée au soutien de l'éducation monolingue en gaélique.

14. R. B. Lepage, A. Tabouret-Keller (1985, p. 236 et 241). 
source fournie par le système culturel de communication au système politique de régulation, ressource à la fois pratique et symbolique ${ }^{15}$. Or ces deux aspects communicatif et symbolique de la langue émergent difficilement dans une situation d'activisme linguistique, a fortiori de restauration linguistique, où la dimension symbolique de la langue est prépondérante et fortement conscientisée.

\section{Une controverse exemplaire}

La négociation de l'exposition publique du gaélique en des territoires étrangers ou non attribués révèle particulièrement le potentiel symbolique de cette langue. L'évènement suivant, survenu lors de l'enquête, en offre une illustration. Le 18 aout 1997, les enseignes bilingues (anglais-gaélique) ont été retirées des murs de la Student's Union ${ }^{16}$ de Queen's University of Belfast. Ce geste a engendré une vaste controverse, dont la présentation permet d'esquisser diverses positions ${ }^{17}$ impliquées dans la dynamique de représentation du gaélique.

Suite à l'adoption de la politique bilingue par le département culturel de l'université en 1986, le gaélique a été ajouté aux inscriptions anglaises sur les panneaux indicateurs de la Student's Union. L'idéologie sous-jacente à cette nouvelle politique était empreinte de pluralisme culturel: l'installation de ces enseignes et l'ouverture d'un certain nombre de cours de langues gratuits avaient pour fonction de traduire la reconnaissance de la diversité culturelle de

15. J.-W. Lapierre (1988, p. 257).

16. La Student's Union est un lieu qui rassemble syndicats, assistance juridique, clubs de sport, magasins, cafétéria, bars, à l'attention des étudiants.

17. Nous faisons référence aux articles suivants: a) "QUB signs move 'fair enough'» (« Retrait des enseignes de l'université de Belfast; d'accord»), Belfast Telegraph, 19 aout 1997, page de garde; b) «Irish activist accuses Union of ending bilingual policy» («Les activistes irlandais accusent l'union estudiantine de mettre un terme à la politique bilingue »), Irish News, 21 aout 1997, p. 21 ; c) «Signs of twisted thinking» («Signes d'une pensée pervertie»), An Phoblacht, 21 aout 1997, p. 8; d) «Symbols reflect our segregated mindsets» («Les symboles reflètent notre mentalité divisée»), Irish News, 25 aout 1997, Viewpoint, p. 6; e) «The real language lovers have lost out to the bigots» («Les véritables amoureux du gaélique ont perdu du terrain face aux bigots»), « The Irish language movement is allied to separatism» ("Le mouvement pour la langue gaélique est associé au séparatisme »), « Dropped signs fuel equality debate» («Les enseignes abandonnées alimentent le débat sur l'égalité»), Irish News, 25 aout 1997, Agenda, p. 22; f) «Bilingual signs are 'ineffectual'» (« Les enseignes bilingues sont inefficientes»), Anderstown News, 06 sept. 1997, p. 8; g) «Queen's controversy must not obscure the fact that Irish language belongs to us all» («La controverse de l'université ne doit pas obscurcir le fait que la langue gaélique nous appartient à tous ») Belfast Telegraph, 30 sept. 1997, Opinion, p. 10; h) «Student's petition for Irish signs to return» ( Pétition de l'union estudiantine pour le retour des enseignes 
la population estudiantine. Ces principes de base ont à nouveau été largement ratifiés par les étudiants à l'occasion d'un référendum en 1994. Cependant, la décision de retirer les enseignes, revendiquée longuement par la Ulster Unionist Youth, est survenue à la suite d'une recommandation de la Fair Employment Commission (FEC) qui considérait que le retrait de ces enseignes bilingues allait créer une atmosphère plus harmonieuse. La présence du gaélique a été identifiée comme l'un des facteurs qui retenaient certains étudiants protestants de quérir des emplois par l'intermédiaire de la Student's Union.

L'université s'est engagée en contrepartie à créer un bureau destiné exclusivement à la bilingual policy, ce qui n'existait pas jusqu'alors. Les partis politiques principaux de la province ont immédiatement pris position sur la décision de supprimer ces enseignes:

- Le UUP (Ulster Unionist Party), voyant dans ce geste la récompense d'une longue campagne contre une idéologie écrasante et dangereuse, a rappelé qu'il s'était toujours opposé à ces enseignes.

- Le DUP (Democratic Unionist Party), de tendance plus extrême, a saisi l'occasion de cet évènement pour réclamer la restauration de l'hymne national britannique God save the Queen lors des cérémonies de remise de diplômes.

- Le SF (Sinn Féin) a condamné le retrait de ces enseignes (a) et décrit ce geste comme une défaite pour la tolérance $(\mathrm{h})$.

- Le SDLP (Social Democratic and Labour Party), au profil nationaliste plus modéré, a porté essentiellement sa critique sur le fait que les étudiants, absents, n'aient pas été consultés, plaçant ainsi la question au niveau de la problématique plus large de la représentativité. En conséquence, ce parti, qui proposait un référendum au sein de l'université, a fait circuler une pétition qui inscrivait ce retrait dans le débat politique et culturel global mené au sein du parlement provisoire de Stormont (h).

Parallèlement, ce débat politique a incité diverses prises de position dans l'opinion publique. Longuement commentée par le biais d'interventions radiophoniques, d'éditoriaux et de courriers de lecteurs, la controverse portait ici sur la question de l'exploitation symbolique du gaélique. Bien que l'espace universitaire soit généralement considéré comme un territoire neutre, il se trouvait soudain identifiable à un microcosme de tout le conflit de la province (g). La question de la neutralité potentielle de la langue a alors soulevé un débat passionné. Constatation faite que cet évènement reflétait la façon dont le

gaéliques»), Irish News, 30 sept. 1997; i) «Queen's signs move defended by chief» («Le déplacement des enseignes de l'université défendu par le chef»), Belfast Telegraph, $1^{\text {er }}$ oct. 1997 ; j) «Trilingual approach is best solution» («L'approche trilingue est la meilleure solution »), Belfast Telegraph, 14 oct. 1997. 
gaélique était utilisé comme outil politique (e), il s'agissait d'évaluer la légitimité de cette association. Ceux qui voyaient dans les connotations politicoreligieuses décernées à la langue une réduction injurieuse mentionnaient régulièrement les quelques exemples de visibilité non problématique du gaélique dans la société, à savoir la présence du terme Failte (Bienvenue) dans l'aéroport international de Belfast et l'inscription Erinn go brach («Irlande pour toujours ») qui figure sur la médaille de cérémonie du maire de la ville depuis 1892.

Diverses mesures ont été proposées pour stabiliser la situation. Ceux qui défendaient la neutralité du gaélique revendiquaient alternativement la restauration des enseignes bilingues ou l'ajout éventuel de l'Ulster-Scots, dialecte implanté sur le littoral par les colons écossais au $16^{\mathrm{e}}$ siècle (e). Pour les autres, il semblait logique que les enseignes ne puissent être remises en place, tant que le gaélique était perçu par certains comme aliénant (d). Il a enfin été suggéré que de nombreuses langues soient utilisées, à l'image de l'internationalité de la population estudiantine concernée, de sorte à désenclaver cette question de toute réflexion provinciale et à rendre à ces panneaux leur statut fonctionnel, leur rôle d'indicateurs.

Entretemps, quelques étudiants ont mené une campagne d'affichage sauvage, couvrant les murs d'inscriptions gaéliques. Toutes les portes des toilettes se voyaient par exemple munies d'inscriptions gaéliques qui se trouvaient arrachées le lendemain ou couvertes d'injures par des adversaires anonymes. Cette réaction au sein du bâtiment a suscité en retour, et une fois encore en vertu de l'analogie que ces réactions évoquaient face au conflit de la province, divers commentaires oscillant entre encouragements et vive inquiétude. Ainsi, cette controverse donne l'occasion de mettre en relief les diverses dimensions relatives à l'exposition du gaélique dans la sphère publique, soit la définition de sa valeur intrinsèque, historique et communicative, face à son statut symbolique, ses connotations identitaires et politiques.

Par ailleurs, le retrait de ces enseignes a naturellement provoqué de fortes réactions parmi les militants pour la promotion du gaélique. Le commentaire de l'éditeur du journal Lá, militant linguistique forcené, offre une synthèse des enjeux perçus par les language fighters dans l'exposition du gaélique. Son argumentation inscrit la question du retrait de ces enseignes dans la problématique de l'inégalité culturelle et des droits relatifs aux langues minoritaires ${ }^{18}$ :

Le gaélique est une langue minoritaire. Or, la visibilité d'une langue est reconnue comme principe fondamental du processus de promotion et de normalisation de

18. Nous traduisons l'article (c) «Signs of twisted thinking», en le synthétisant. 
toute langue minoritaire. Dès lors, l'absence du gaélique dans la sphère publique équivaut à un acte discriminatoire envers une minorité, ceci étant mis en rapport avec les discriminations raciale et sexuelle. Les locuteurs celtophones forment une communauté extrêmement diversifiée. Par conséquent, l'association exclusive de la langue à la communauté catholique est une simplification injurieuse. L'argument selon lequel l'absence de gaélique est une condition nécessaire à la neutralité de l'environnement est tout simplement incompréhensible, le bilinguisme n'étant pas en soi exclusif. Dès lors, ce geste ne s'explique que par une idéologie délibérément ségrégationniste. Par l'intermédiaire de cet acte d'intolérance, les instigateurs de ce retrait, la FEC particulièrement, qui sont officiellement dédiés au principe d'égalité, se révèlent trompeurs. Au même titre, le gouvernement, qui prétend représenter un État démocratique engagé dans un processus de valorisation de la diversité culturelle, devrait saisir cette occasion pour agir à l'encontre de ces attitudes incohérentes. Il est rappelé que le traitement du gaélique en Irlande du Nord, qui ne bénéficie d'aucune reconnaissance institutionnelle comparable à celles du gallois et de l'écossais, est dissonant au sein de l'Union européenne. Il est donc dans l'intérêt du gouvernement de s'aligner sur le comportement généralement favorable des pays qui l'entourent, d'accorder un statut constitutionnel au gaélique et de promouvoir sa diffusion par le biais de l'éducation et des médias. Par conséquent, le militantisme demeure légitime; cet évènement révèle en somme la nécessité d'une lutte pour une représentation linguistique équitable.

Cette argumentation en faveur de l'activisme linguistique est essentiellement exprimée dans le registre des droits. Ceci nous semble pouvoir être interprété en termes de territorialité à double titre. D'une part, cette position traduit une conception du droit de nature philosophique, faisant appel au droit individuel à l'identité et à la différence, soit au principe de personnalité, qui s'oppose au principe de territorialité, propre à toute gestion politique caractérisée par la primauté de l'État sur l'individu ${ }^{19}$. Ces deux pôles engendrent un continuum sur lequel s'inscrivent les politiques d'aménagement linguistique, et où prévalent alternativement les concepts de droit territorial, droit individuel et droit collectif ${ }^{20}$. Or ce commentaire semble bien comporter la reconnaissance du pouvoir de la majorité, mais la négation du principe cujus regio ejus lin$g u a^{21}$. D'autre part, si l'on considère la capacité de balisage symbolique propre au gaélique dans le reste de la ville, la présence du bilinguisme dans ce contexte dit neutre équivaut à la reconnaissance d'un partage de ce territoire. L'on peut alors concevoir que cette accusation de linguicisme exprime fondamentalement une défaite territoriale. Ainsi, cet événement, bien qu'anecdotique, met en relief le type de négociation dont peut faire l'objet la visibilité du

19. W. F. Mackey (1989).

20. Voir C. B. Paulston (1997).

21. B. Py (1989, p. 10). 
gaélique. En outre, cette controverse illustre la manière intéressante dont une polémique relative au bilinguisme se superpose aux dimensions identitaire et territoriale.

Lactivisme linguistique en Irlande du Nord prend place dans un milieu urbain dont la configuration spatiale reflète la division de la société globale. Comme le relève G. Maguire, il est en fin de compte peu surprenant que la configuration unique de la ville de Belfast, avec ses ghettos et son degré de conscience politique et culturelle extrêmement élevé, ait produit une approche radicale de la restauration linguistique ${ }^{22}$. Le fait que cette logique de territorialisation impliquant la langue se produise sur une ile est sans doute également significatif en termes d'appropriation symbolique, si l'on considère que "l'une des originalités insulaires réside dans la densité du processus de sacralisation de l'espace», et que "l'esprit de groupe, le "nous" se constitue plus facilement dans un univers protégé ${ }^{23}$. Dans ce contexte, le gaélique, emblématisé, sert de balise territoriale. Il fait partie des ressources symboliques de la communauté catholique minoritaire. Il est utilisé pour marquer les limites de son territoire et est également perçu comme représentant cette communauté dans les autres territoires de la ville ${ }^{24}$. Toutefois, comme l'illustre le débat au sujet des enseignes bilingues, le statut de cette langue fait l'objet de multiples interprétations et de négociations, qui sont notamment influencées par l'évolution des politiques culturelles menées au sein de l'Union européenne.

\section{Bibliographie}

CENTLIVRES P., 1986, Les sciences sociales face à l'identité régionale, Berne-Stuttgart, P. Haupt, $280 \mathrm{p}$.

HOBSBAWN E., 1992, «Ethnicity and nationalism in Europe today», Anthropology today, $\mathrm{n}^{\circ} 8$ (1), Oxford, p. 1-8.

HOBSBAWN É., 1992, Nations et nationalisme depuis 1780, Paris, Gallimard, 254 p.

LAPIERRE J.-W., 1988, Le pouvoir politique et les langues : Babel et Léviathan, Paris, PUF, $295 \mathrm{p}$.

22. G. Maguire (1991, p. 14).

23. F. Péron (1993, p. 155-156). Voir aussi F. Thual, qui pose comme hypothèse que l'insularité et son exigüité territoriale sont des facteurs de condensation des conflits identitaires (1995, chap. 15).

24. Ces caractéristiques distinguent le type de mobilisation identitaire à l'œuvre autour $\mathrm{du}$ gaélique irlandais d'autres formations de militantisme linguistique européennes, telles que par exemple les mouvements écossais, basque, catalan, lombard ou breton. 
LEPAGE R. B., TABOURET-KELLER A., 1985, Acts of identity, Cambridge, Cambridge University Press, $275 \mathrm{p}$.

LOSONCZY A.-M., 1997, «Les itinéraires de la patrie; de la construction de l'espace interpatriotique à propos de la Hongrie contemporaine», Dire les autres; réflexions et pratiques ethnologiques, Lausanne, Payot, p. 177-195.

LÜDI G., PY B., 1995, Changement du langage et langage du changement; aspects linguistiques de la migration interne en Suisse, Lausanne, L'Âge d'Homme, 301 p.

MACKEY W. F., 1989, «L'irrédentisme linguistique: une enquête témoin », Plurilinguisme: normes, situations, stratégies, Paris, L'Harmattan, p. 257-284.

MAGUIRE G., 1991, Our Own Language: An Irish Initiative, Clevedon, Multilingual Matters, $\mathrm{n}^{\circ} 66,187 \mathrm{p}$.

MILROY L., 1989, Observing and Analysing Natural Languages. A Critical Account of Sociolinguistic Method, Oxford, Basil Blackwell Ltd, 230 p.

MOLINER P., 1996, Images et représentations sociales, Grenoble, PUG, 275 p.

MÜLLER V., 1999, La défense du gaélique à Belfast: approche ethnologique d'un mouvement linguistique en contexte européen, Neuchâtel, Institut d'ethnologie, $105 \mathrm{p}$.

PAULSTON C. B., 1997, «Language policies and language rights », Annual Review of Anthropology, $n^{\circ}$ 26, p. 73-85.

PÉRON F., 1993, Des iles et des hommes, Rennes, Éditions de la Cité, 287 p.

POUTIGNAT P., STREIFF-FENART J., 1995, Théories de l'ethnicité, Paris, PUF, $270 \mathrm{p}$.

PY B., 1989, Minorisation linguistique et interaction, Genève, Librairie Droz, 232 p.

RUANNE J., TODD J., 1996, The Dynamics of Conflict in Northern Ireland: Power, Conflict and Emancipation, Cambridge, Cambridge University Press, 365 p.

SKUTNABB-KANGAS T., 1990, Language, Literacy and Minorities, London, MRG Report, $34 \mathrm{p}$.

THUA F., 1995, Les conflits identitaires, Paris, Ellipses, 191 p. 